\title{
Impact of community pharmacist intervention on concurrent benzodiazepine and opioid prescribing patterns
}

\author{
Georgia G. Luchen*, Emily S. Prohaska, Janelle F. Ruisinger, Brittany L. Melton
}

\section{A R T I C L E I N F O}

\section{Article history:}

Received 13 June 2018

Accepted 7 October 2018

Available online 11 December 2018

\begin{abstract}
A B S T R A C T
Objectives: (1) To evaluate the number of opioid/benzodiazepine (BZD) prescription changes resulting from pharmacist communication to prescriber(s); (2) to determine the number of patients on concurrent opioid/BZD therapy from single versus multiple prescribers; (3) to compare the number of opioid/BZD prescription changes resulting from communication when a single versus multiple prescribers was involved in a patient's care; and (4) to compare the number of opioid/BZD prescription changes resulting from communication via fax versus the Kansas Health Information Network (KHIN) direct messaging feature.

Methods: Prospective study conducted at 13 community pharmacies, including patients 18 years of age or older simultaneously filling opioid and BZD prescriptions within 90 days before October 2017. Prescribers received faxed or KHIN communication proposing evidence-based prescription changes to opioid/BZD agents. Prescription changes were evaluated weekly for 3 months after the initial intervention. Descriptive statistics assessed demographics and the number and types of prescription changes. Spearman rho correlations compared prescription changes and number of prescriptions to number of prescribers; a priori alpha was set at 0.05 . Results: A total of 137 prescribers and 121 patients were included. Ninety-nine prescribers were contacted via fax and 38 via KHIN. After 4 weeks, 34 recommendations were received: 20 responses (59\%) indicated rejection of recommendations, 5 (15\%) approved BZD taper/ discontinuation, $3(9 \%)$ deferred changes until a patient visit, 2 (6\%) approved opioid taper/ discontinuation, $2(6 \%)$ prescribed naloxone, and $2(6 \%)$ withdrew from the patient's care. Three months after communication, 35 prescription changes were noted: 22 (63\%) opioid/BZD agent tapers/discontinuation, 14 (26\%) opioid/BZD dose increases, and 2 (6\%) naloxone prescriptions. There was positive correlation between the number of tapered/discontinued agents and the number of prescribers involved in a patient's care $(P=0.046)$.

Conclusion: A faxed pharmacist intervention may help to reduce opioid/BZD coprescribing, especially when multiple providers are involved in a patient's care.
\end{abstract}

(C) 2019 American Pharmacists Association ${ }^{\circledR}$. Published by Elsevier Inc. All rights reserved.
Drug misuse or abuse is a critical issue in the United States, leading to steady increases in emergency department (ED) visits. $^{1}$ Opioid overdose deaths doubled from 2015 to 2016 from 3.1 to 6.2 per 100,000 people. $^{2}$ Moreover, opioids and benzodiazepines (BZDs), used alone or in combination, have

Disclosures: None of the authors report a potential conflict of interest. Funding: American Pharmacists Association Foundation (Incentive Grant, 2018). The funding source did not have any role in research conduction or article preparation.

Previous presentations: American Pharmacists Association Annual Meeting and Exposition, Nashville, TN, March 2018; Midwest Pharmacy Residents Conference, Omaha, NE, May 2018.

* Correspondence: Georgia G. Luchen, Balls Food Stores, 13600 S. Blackbob Rd., Olathe, KS 66062.

E-mail address: gina.galanou@gmail.com (G.G. Luchen). been identified as the most common pharmaceuticals involved in drug-related deaths. ${ }^{3}$

To address the issue of drug misuse, and to aid clinicians in the prescribing of high-risk medications, the Centers for Disease Control and Prevention (CDC) published an evidencebased guideline on opioid prescribing for chronic pain in $2016 .{ }^{4}$ The guideline outlines the risks of coprescribing opioids with BZDs and encourages clinicians to openly discuss the risks of the combination with patients or to avoid coprescribing whenever possible.

Subsequently, the Pharmacy Quality Alliance (PQA) developed several measures involving medications associated with a high risk for overdose. Specifically, new measures address (1) opioids from multiple prescribers and (2) concurrent use of opioids and BZDs. ${ }^{5}$ The pharmacist role in monitoring medication prescribing patterns was also highlighted in a study 
about inappropriate drug combinations in the elderly. Results indicated that a higher number of prescribers was associated with a higher number of drug interactions and suggested that pharmacists could play a role in reducing drug interactions. ${ }^{6}$

Few studies have examined the impact of pharmacist interventions on opioid and BZD coprescribing, and most were in specialized settings. A retrospective study examined the impact of pharmacy consultations on opioid and BZD coprescribing in a veteran population and found a reduction in the coprescribing practices and overdose-related events. ${ }^{7}$ Similarly, a pilot study assessed the impact of a pharmacist previsit consultation on high-risk patients treated with opioids in a family medicine clinic. Using the CDC guidelines for pharmacist recommendations, it reduced opioid use and decreased opioid and BZD coprescribing. ${ }^{8}$ Despite some encouraging results regarding pharmacists' impact in reducing coprescribing of opioids and BZDs, to date no studies have been conducted in community pharmacies.

Although community pharmacies may not have direct access to electronic health records (EHRs), tools such as statelevel health information exchange (HIE) programs could facilitate transmission of patient care recommendations. A number of studies evaluated the use of HIEs in the United States, finding that clinicians' perception of benefits of the platform depends on the data availability and workflow integration. ${ }^{9,10}$ The Kansas Health Information Network (KHIN), is regarded as one of the most functional HIEs in the United States, ${ }^{11,12}$ with robust statewide participation that provides a sizeable volume of available patient data from 126 hospitals and $75 \%$ of the state's physician practices. In addition, users, including pharmacists, are able to collaborate with one another via a direct messaging feature. To date, use of the KHIN messaging feature by community pharmacists and providers has not been studied. Currently, Balls Food Stores (BFS) community pharmacists use faxing as a standard interprofessional communication tool concerning medication recommendations that are not of immediate concern.

The present study sought to examine the impact of pharmacist intervention on reducing opioid and BZD coprescribing and to evaluate the comparative effectiveness of tools designed to facilitate interdisciplinary communications.

\section{Objectives}

The objectives were to (1) evaluate the number of opioid/ BZD prescription changes resulting from pharmacist communication to the prescriber(s); (2) determine the number of patients on concurrent opioid/BZD therapy from single versus multiple prescribers; (3) compare the number of opioid/BZD prescription changes resulting from communication when a single versus multiple prescribers was involved in a patient's care; (4) compare the number of opioid/BZD prescription changes resulting from communication via fax versus the KHIN messaging feature.

\section{Methods}

This prospective study used dispensing records from 13 Kansas pharmacies within BFS, a supermarket chain in the Kansas City metropolitan area.
Eligible patients were 18 years of age or older and had received 1 or more oral opioid and BZD agents in the 90 days before October 1, 2017. Inclusion and exclusion criteria were derived from the PQA measure related to concurrent use of opioids and BZDs. ${ }^{5}$ Included participants had concurrent active prescriptions for opioids and BZDs for 30 or more cumulative days in the past 90 days. Patients receiving hospice care or who had cancer-related diagnoses were excluded. Prescriber and patient data, including demographics and prescription data, were collected from the patient's dispensing record by a single pharmacist and verified by a second pharmacist for accuracy. Discrepancies were discussed and subsequently corrected.

After identifying the study sample, patients' prescribers were contacted if they had prescribed an opioid or BZD agent for a supply of 7 or more days. The 7-day supply limitation was implemented to avoid contacting prescribers not regularly involved in the patient's care and those who prescribed opioids only for acute pain management. ${ }^{4}$

Both the fax and KHIN communications included identical written information comprising (1) a letter introducing the study purpose and relevant clinical guidelines; (2) patient dispensing records, including the name, dose, and days' supply of the last 3 fills for each opioid and BZD agent, as well as names of other prescribers who had prescribed any opioid or BZD agents; and (3) a set of standardized recommendations. The prescriber was asked to select 1 or more of the suggested recommendations and return the response to the pharmacy. The recommendations were derived from the $\mathrm{CDC}$ guidelines for prescribing opioids for chronic pain and included options for tapering or discontinuing the opioid or BZD agent(s), replacing the agent(s), prescribing naloxone, declining any action, or providing alternate suggestions or comments. ${ }^{4}$

Communications were sent once per week, for up to 3 weeks, until a response was received. The methods of communication were not altered after stratification, even if no responses were received, to ensure that data would be an accurate reflection of the fax or KHIN effectiveness. Faxed responses were collected from each participating pharmacy at 1-week intervals after the communication delivery to allow time for prescribers to respond to the request. Electronic responses from KHIN were collected at the same time as fax responses via platform access. Three months after the initial communication, dispensing records of each study patient were reviewed to record medication changes related to opioid or BZD agents or receipt of a naloxone prescription. This pharmacy records review ensured that all relevant prescription changes were captured, even if a response to the standardized recommendations was not directly communicated by the prescriber. The 3-month time frame was selected because Kansas regulations allow up to a 3-month supply on any schedule II prescription. A positive medication change was defined as tapering or discontinuing an opioid or BZD agent.

Data analysis was conducted with the use of SPSS v. 24. Descriptive statistics were used to assess patient and provider demographics and determine the number and types of prescription changes. Chi-square was used to compare demographics for KHIN versus non-KHIN providers. Spearman rho correlations compared the number of prescription changes with the number of providers involved in the patient's care, as well as the number of prescriptions per patient with the 
Table 1

Demographics of patients, included in the study owing to concurrent use of opioid and benzodiazepine (BZD) agents, and prescribers involved in the patients' care, n (\%)

\begin{tabular}{|c|c|c|}
\hline \multicolumn{2}{|c|}{ Patient parameters $(\mathrm{n}=121)$} & \\
\hline Female sex & $81(67)$ & \\
\hline Age (y), median (IQR) & $62(52-69.5)$ & \\
\hline Had single prescriber & $69(57)$ & \\
\hline \multirow{2}{*}{$\begin{array}{l}\text { Prescriber parameters } \\
(\mathrm{n}=137)\end{array}$} & \multicolumn{2}{|l|}{ Active KHIN account } \\
\hline & Yes $(\mathrm{n}=38 ; 28 \%)$ & No $(n=99 ; 72 \%)$ \\
\hline \multicolumn{3}{|l|}{ Sex } \\
\hline Female $^{\mathrm{a}}$ & $20(53)$ & $27(27)$ \\
\hline Male & $18(47)$ & $72(73)$ \\
\hline \multicolumn{3}{|l|}{ Prescriber type } \\
\hline Physician prescriber & $35(92)$ & $87(88)$ \\
\hline Mid-level prescriber & $3(8)$ & $12(12)$ \\
\hline
\end{tabular}

Abbreviations used: IQR, interquartile range; KHIN, Kansas Health Information Network.

a Statistically significant difference $(P=0.005)$.

number of prescribers involved in the patient's care. Statistical significance was defined as $P \leq 0.05$.

This study was granted exempt status from the University of Kansas Medical Center Human Research Protection Program.

\section{Results}

A study population of 3974 unique patients was identified. One hundred twenty-two patients met the inclusion criteria, and 1 patient was excluded owing to a cancer diagnosis. Patients received 1 or more prescriptions from 149 unique prescribers during the study period.

After limiting contact to those who had prescribed more than a 7-day supply of either agent, 137 prescribers were included in the study. Of these, 38 had an active KHIN account and were contacted via the KHIN secure messaging feature. The remaining 99 prescribers were contacted via fax. Seventeen of the 38 KHIN messages failed to be delivered; KHIN technical support was unable to provide resolution for delivery failure. Demographics for both patients and prescribers are presented in Table 1.

A total of 32 prescribers responded to the pharmacist's faxed communication during the initial 4-week period, providing 34 unique responses ( 1 prescriber made 2 recommendations for 1 patient, and another made recommendations for 2 separate patients). Details regarding the number and type of responses received each week following the pharmacist communication are presented in Table 2.

The pharmacy records review that occurred 3 months after the intervention identified 35 prescription changes, corresponding to 29 of 122 unique patients. One or more positive changes were observed for 19 unique study patients (16\%), and a dose increase of either the opioid or BZD agent was observed for 9 unique patients (7\%). A request for clinical rationale was not elicited from each prescriber, although some prescribers volunteered information. One prescriber indicated that the BZD agent was prescribed for seizures, warranting the BZD dose increase. In 2 patients the prescriber tapered or discontinued the opioid but increased the BZD dose. A detailed description of provider responses and prescription changes is presented in Table 2. No responses were received from prescribers contacted via KHIN; however, 4 prescription changes were observed at the 3-month follow-up. Three changes were positive, and the other was an opioid dose increase.

There was a statistically significant positive correlation between the number of positive prescription changes and the number of prescribers involved in a patient's care, indicating that more positive changes occurred when multiple prescribers were involved in a patient's care $(r=0.182 ; P=0.046)$. In addition, a higher number of opioid or BZD prescriptions were observed when more than 1 prescriber was involved in the patient's care $(r=0.386,0.325$, and 0.391 , respectively for opioids, BZD, and total prescriptions; $P<0.001$ ).

\section{Discussion}

Pharmacists are increasingly called on to address the opioid epidemic and reduce opioid/BZD coprescribing. ${ }^{5}$ Little is known about community pharmacist impact on opioid prescribing trends or the best methods for interdisciplinary communication. To the authors' knowledge, this is the first study to examine multiple communication approaches to influence opioid and BZD prescribing patterns in the community pharmacy setting.

In this study, the majority of prescription changes (63\%) that occurred after pharmacist intervention resulted in tapering or discontinuation of the opioid or BZD agent. These observations are consistent, though less marked, with findings from research conducted in different practice settings. ${ }^{7,8}$ Pardo et al. found a 35\% reduction in opioid and BZD coprescribing after implementation of a prior authorization pharmacist consult in the veteran population, ${ }^{7}$ compared with $16 \%$ in the present study. Notably, pharmacists in the Pardo et al. study were integrated in the Veterans Affairs Health Care System, and prescribers were required to acknowledge the pharmacist consultation before sending the prescription. In contrast, there was no way to verify receipt of the communication provided in the present study. In addition, BFS pharmacists were not contractually affiliated with any of the prescribers contacted, possibly contributing to the lower observed response rate.

Reduction in coprescribing of opioids and BZDs was also observed in another study performed at a family medicine residency clinic. ${ }^{8}$ Clinic pharmacists reviewed patient charts before the prescriber visits and documented recommendations in the patient's EHR. Similarly to the present study, in addition to the opioid/BZD coprescribing reduction, pharmacists also focused on recommending naloxone initiation. A total of 37 naloxone prescriptions were recommended by clinic pharmacists, and 20 were offered by the prescribers. In our study, naloxone was recommended as an option for all patients, but only 2 prescriptions were furnished. The lower number of naloxone prescriptions dispensed in this study compared with the one conducted by Cox et al. could be explained by the fact that in Cox's study, pharmacists provided individualized recommendations within the patient's EHR, which were available during the patient's visit with the prescriber. in contrast, the present study encouraged naloxone prescribing as part of a standardized list. As a result, prescribers did not have immediate electronic access to the community pharmacist's recommendation during the patient visit. Furthermore, this study did not have a method of 
Opioid and benzodiazepine co-prescribing patterns

Table 2

Type and number (\%) of prescriber responses and prescription changes that occurred after the pharmacist communication

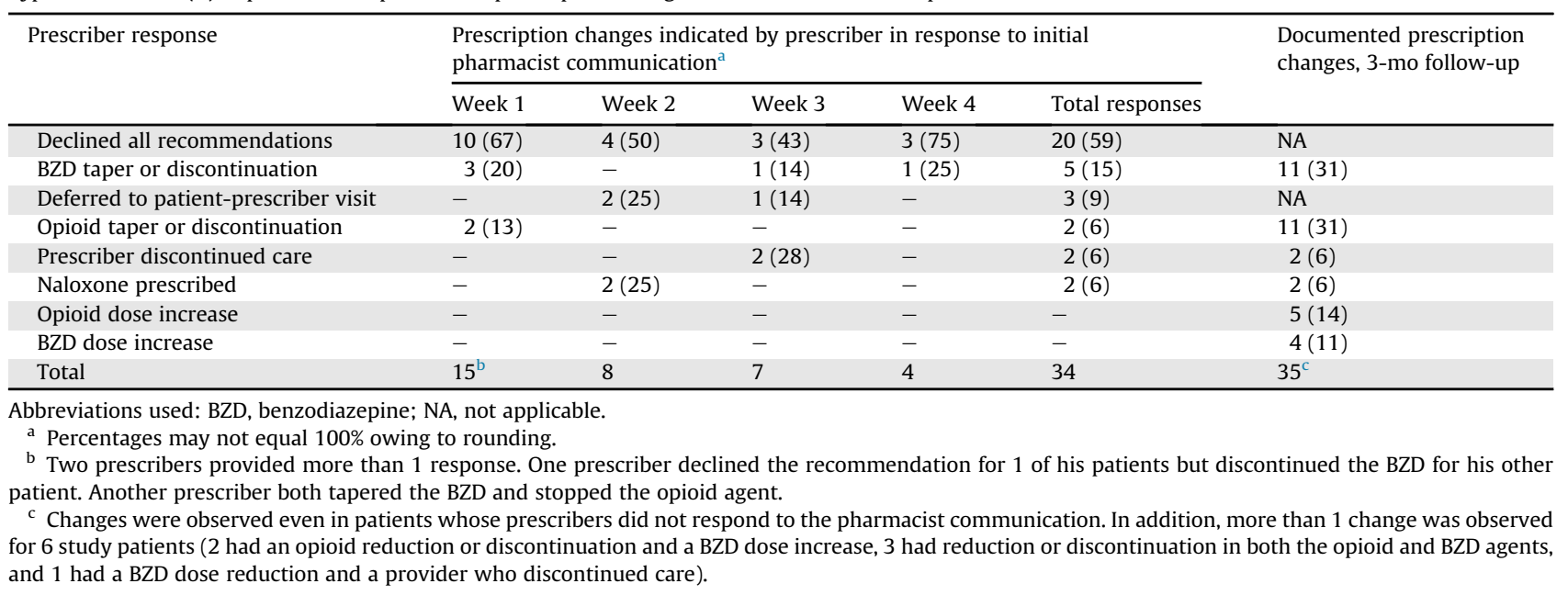

verifying if the prescriber offered naloxone and the patient declined.

The positive correlation between the number of prescription changes and number of prescribers involved in a patient's care highlights the role of community pharmacists in helping promote transparency between providers, especially when care is disjointed. Specifically, 2 prescribers chose to discontinue care for the patient and defer to an alternate provider, revealing they may have been unaware of the patient's full medication history or existence of additional prescribers. The literature suggests that opioid prescribing by multiple prescribers leads to higher hospitalization risks ${ }^{13}$ and that collaboration between providers could improve coordination of care. ${ }^{14}$ By raising awareness and promoting transparency, pharmacists could be part of this collaboration, helping to improve patient outcomes.

Finally, one of this study's primary objectives was to compare the number of prescription changes resulting from communications sent via fax versus KHIN messaging. This comparison was not possible, because no responses were received from the KHIN prescriber cohort, and the number of changes that occurred from KHIN prescribers at the 3-month follow-up were too few to provide statistical comparison. The low use of KHIN is in line with previous studies. A systematic review examining HIE use among health professionals found that although HIE use has grown in the past 10 years, it is primarily adopted by larger health systems. In addition, comparisons among health professionals showed a greater use of HIE by nurses and ED physicians. ${ }^{15}$ The present study's findings suggest that HIE use by individual prescribers for interprofessional multi-institutional communication may be more limited compared with internal health system use.

Overall, the study results align with the idea that community pharmacists could influence opioid and BZD coprescribing patterns, and they support faxing as a useful interprofessional communication tool. Future studies examining a multimodal approach (e.g., fax followed by a telephone call) for communicating interventions could be helpful in identifying more effective methods for interprofessional collaboration. Finally, studies involving a control arm without a pharmacist intervention could help to clarify which findings directly resulted from the pharmacist communication.

\section{Limitations}

There were several limitations to this study. First, only oral medications were studied. Second, the limited number of providers with active KHIN accounts did not allow for study randomization regarding intervention communication media. Third, pilot messages were not sent to providers before the study to ensure KHIN functionality, and when KHIN or fax communications were sent, there was no method to confirm if prescribers received the message. Fourth, the study used the HIE platform unique to Kansas, so the findings may have limited generalizability. Fifth, the study was conducted in a short time frame, limiting the sample size. Sixth, fills at nonstudy pharmacies were not examined. Finally, our study did not include a control group, which could demonstrate whether similar opioid and BZD prescription changes would occur without a pharmacist intervention.

\section{Conclusion}

A faxed intervention from a community pharmacist may help to reduce opioid/BZD coprescribing, especially when multiple providers are involved in a patient's care. In addition, KHIN secure messaging was not found to be an effective medium for communicating patient care recommendations from the community pharmacy setting at this time.

\section{References}

1. Center for Behavioral Health Statistics and Quality, Substance Abuse and Mental Health Services Administration. Drug Abuse Warning Network, 2010: national estimates of drug-related emergency department visits. Available at: https://www.samhsa.gov/data/sites/default/files/DAWN2k1 OED/DAWN2k10ED/DAWN2k10ED.htm. Accessed August 25, 2017.

2. Hedegaard H, Warner M, Miniño AM. Drug overdose deaths in the United States, 1999-2016. Available at: https://www.cdc.gov/nchs/data/databriefs/ db273.pdf. Accessed August 28, 2017.

3. Jones CM, Mack KA, Paulozzi LJ. Pharmaceutical overdose deaths, United States, 2010. JAMA. 2013;309:657-659. 
4. Dowell D, Haegerich TM, Chou R. CDC guideline for prescribing opioids for chronic pain. MMWR Recomm Rep. 2016;65(1):1-49.

5. Pharmacy Quality Alliance. PQA performance measures. Available at: http://pqaalliance.org/measures/default.asp. Accessed August 18, 2018.

6. Tamblyn RM, McLeod PJ, Abrahamowicz M, et al. Do too many cooks spoil the broth? Multiple physician involvement in medical management of elderly patients and potentially inappropriate drug combinations. CMAJ. 1996;154(8):1177-1184.

7. Pardo D, Miller L, Chiulli D. Implementation of a pharmacy consult to reduce co-prescribing of opioids and benzodiazepines in a veteran population. Subst Abus. 2017;38(2):157-160.

8. Cox N, Tak CR, Cochella SE, Leishman E, Gunning K. Impact of pharmacist previsit input to providers on chronic opioid prescribing safety. J Am Board Fam Med. 2018;31(1):105-112.

9. Hincapie AL, Warholak TL, Murcko AC, Slack M, Malone DC. Physicians opinions of a health information exchange. $J$ Am Med Inform Assoc. 2011;18(1):60-65.

10. Rudin R, Volk L, Simon S, Bates D. What affects clinicians' usage of health information exchange? Appl Clin Inform. 2011;2(3):250-262.

11. Black Book Market Research 2015 user survey: state of the enterprise health information exchange industry. Available at: https://blackbook marketresearch.com/uploads/pdf/2015-Black-Book-State-of-the-EnterpriseHIE-Industry-Report-VENDOR-REVIEW-ONLY.pdf. Accessed July 27, 2018.
12. Hartford Business. CT health information exchange efforts forge ahead. Available at: http://www.hartfordbusiness.com/article/20170626/PRINT EDITION/306229937/ct-health-information-exchange-efforts-forge-ahead. Accessed July 27, 2018.

13. Jena AB, Goldman D, Schaeffer LD, Weaver L, Karaca-Mandic P. Opioid prescribing by multiple providers in Medicare: retrospective observational study of insurance claims. BMJ. 2014;348:1393.

14. Ong M-S, Olson KL, Cami A, et al. Provider patient-sharing networks and multiple-provider prescribing of benzodiazepines. J Gen Intern Med. 2016;31(2):164-171.

15. Devine EB, Totten AM, Gorman P, et al. Health information exchange use (1990-2015): a systematic review. EGEMS (Wash DC). 2017;5(1):27.

Georgia G. Luchen, PharmD, PGY-1 Community-Based Pharmacy Resident, Balls Food Stores, Olathe, KS, and the University of Kansas School of Pharmacy, Lawrence, KS

Emily S. Prohaska, PharmD, BCACP, BCGP, Pharmacy Clinical Services Coordinator, Balls Food Stores, Olathe, KS

Janelle F. Ruisinger, PharmD, FAPhA, Clinical Professor, PGY-1 CommunityBased Residency Director, Department of Pharmacy Practice, University of Kansas School of Pharmacy, Lawrence, KS

Brittany L. Melton, PharmD, PhD, Assistant Professor, Department of Pharmacy Practice, University of Kansas School of Pharmacy, Lawrence, KS 